\title{
Lipoxin A4 protects against paraquat-induced acute lung injury by inhibiting the TLR4/MyD88-mediated activation of the NF-kB and PI3K/AKT pathways
}

\author{
YUHUA LI $^{1}$, NA WANG $^{1,2}$, ZHONGLIANG MA $^{1}$, YUNWEN WANG $^{1}$, \\ YUAN YUAN $^{1}$, ZHITAO ZHONG $^{1}$, YI HONG ${ }^{1}$ and MIN ZHAO $^{1}$ \\ ${ }^{1}$ Department of Emergency Medicine, Shengjing Hospital of China Medical University; \\ ${ }^{2}$ Occupational Disease and Occupational Health Prevention and Control Institute, \\ Liaoning Center for Disease Control and Prevention, Shenyang, Liaoning 110004, P.R. China
}

Received December 24, 2020; Accepted February 26, 2021

DOI: 10.3892/ijmm.2021.4919

\begin{abstract}
Paraquat (PQ) causes serious oxidative stress and inflammatory responses, particularly to the lungs. Since lipoxin A4 (LXA4) functions as an anti-inflammatory mediator, the present study aimed to explore its effects on PQ-induced acute lung injury (ALI) and to elucidate the possible underlying mechanisms. PQ was administered to male SD rats and RAW264.7 cells to establish a model of poisoning, and LXA4 was used as an intervention drug. LXA4 treatment attenuated PQ-induced lung injury, and this was accompanied by decreased tumor necrosis factor (TNF)- $\alpha$ and interleukin (IL)- $1 \beta$ secretion levels, and reduced oxidative stress damage. Additionally, LXA4 treatment inhibited the activation of the inflammation-related signaling molecules, Toll-like receptor 4 (TLR4), myeloid differentiation primary response 88 (MyD88), nuclear factor (NF)- $\mathrm{B}$ p 65 , p-phosphoinositide 3-kinase (PI3K) and $\mathrm{p}$-AKT. Furthermore, the in vitro experiments further confirmed that the beneficial effects of LXA4 on PQ-induced damage were TLR4-dependent. Hence, the present study demonstrated that LXA4 attenuated PQ-induced toxicity in lung tissue and RAW264.7 macrophages, and that this protective effect may be closely related to the mitigation of inflammatory responses, oxidative stress damage and the TLR4/MyD88-mediated activation of the PI3K/AKT/NF-kB pathway.
\end{abstract}

Correspondence to: Dr Min Zhao, Department of Emergency Medicine, Shengjing Hospital of China Medical University, 36 Sanhao Street, Heping, Shenyang, Liaoning 110004, P.R. China E-mail: zhaom_sj@163.com

Key words: paraquat, acute lung injury, lipoxin A4, Toll-like receptor 4/myeloid differentiation primary response 88 , PI3K/AKT, $\mathrm{NF}-\mathrm{\kappa B}$

\section{Introduction}

Paraquat (PQ) is a highly effective and inexpensive contact heterocyclic herbicide and one of the most widely used pesticides in agriculture globally (1). PQ poisoning has a high mortality rate, with no specific antidote or effective treatment option (2). PQ can cause damage to the lungs, liver, kidney, heart and other organs, with lung damage being the most prominent. Due to the strong dopamine absorption system in the lungs, $\mathrm{PQ}$, which has a chemical structure similar to polyamines, mainly accumulates in the lungs (3). Consequently, PQ poisoning leads to acute lung injury (ALI) or even acute respiratory distress syndrome (ARDS), which is responsible for early death (4). The mechanisms through which PQ causes ALI are complex and unclear; however, the majority of studies have reported that the biological processes of redox response and inflammatory cascade reaction play important roles in PQ poisoning (5-8).

Lipoxins (LXs) are a type of endogenous lipid media produced by arachidonic acid (AA) during sequential catalysis by different lipoxygenases. They can be divided into 4 types according to their molecular conformation (9), among which lipoxin A4 (LXA4) is generated via continuous oxidation catalysis. As a negative regulator of inflammation, LXA4 functions as an anti-inflammatory agent, promoting the resolution of inflammation, regulating immune function, and stimulating tissue and cell damage repair $(10,11)$. In various inflammation-related diseases, LXA4 inhibits the release of pro-inflammatory factors and the infiltration of inflammatory cells, and promotes the chemotaxis and recruitment of macrophages, thus enhancing their non-inflammatory phagocytotic function $(12,13)$. Furthermore, LXA4 inhibits ROS generation, preventing tissues or macrophages from oxidative stress-mediated damage (14-16). LXA4 has also been found to increase the activity of antioxidant enzymes in various organs, and to play a protective role in restoring the oxidant/antioxidant balance (17-19).

However, the biological effects of LXA4 on PQ-induced ALI have yet to be confirmed, at least to the best of our knowledge. Therefore, the aim of the present study was to investigate 
the role of LXA4 in PQ-induced ALI and to elucidate the possible underlying mechanisms.

\section{Materials and methods}

Animals. Specific pathogen-free male SD rats (aged 6-8 weeks; body weight, 250-300 g; $\mathrm{n}=40$ ), purchased from Beijing HFK Bioscience Co. Ltd, were allowed to acclimatize in the laboratory at the Experimental Animal Center of Shengjing Hospital of China Medical University for 1 week with free access to food and drink. The animals were maintained under a controlled temperature $\left(20-25^{\circ} \mathrm{C}\right)$ and constant humidity $(40-70 \%)$, and were subjected to a natural photoperiod (12/12 $\mathrm{h}$ light/dark cycle). The experiments were approved by the Ethics Committee of Shengjing Hospital of China Medical University (approval no. 2019PS656K). All procedures involving animals were performed in accordance with the ARRIVE guidelines and the National Institutes of Health Guidelines for the Care and Use of Laboratory Animals (NIH Publications no. 8023, revised in 1978).

Establishment of animal models. The rats were randomly divided into 4 groups as follows: The control (CON; $n=10), P Q$ poisoning $(\mathrm{PQ} ; \mathrm{n}=10), \mathrm{PQ}$ poisoning + lipoxin $\mathrm{A} 4(\mathrm{PQ}+\mathrm{LXA} 4 ; \mathrm{n}=10)$ and LXA4 $(n=10)$ groups. The rats in the PQ and PQ + LXA4 groups were intraperitoneally injected with $\mathrm{PQ}$ (Sigma-Aldrich; Merck KGaA) at a dose of $20 \mathrm{mg} / \mathrm{kg}(6,20)$, while an equivalent volume of saline was injected into the rats in the CON and LXA4 groups. At $1 \mathrm{~h}$ after the PQ or saline injection, the rats in the PQ + LXA4 and LXA4 groups were injected daily with LXA4 diluted to $0.1 \mathrm{mg} / \mathrm{ml}$ in saline (Cayman Chemical Co.) at a dose of $2 \mu \mathrm{g} / \mathrm{kg}$ through the tail vein $(21,22)$, while the PQ and $\mathrm{CON}$ groups were treated with an injection of equal volume of normal saline. The rats were sacrificed by an overdose of sodium pentobarbital (>150 mg/kg) injected intraperitoneally following exposure to $\mathrm{PQ}$ for $48 \mathrm{~h}$. When the cessation of the heartbeat and breathing of the rats was confirmed, and there were no reflexes, the death of the experimental animals was confirmed painlessly. Subsequently, bronchoalveolar lavage fluid (BALF) and lung specimens were collected.

Analysis of lung wet/dry weight ratio. The upper lobe of the right lung was collected following exposure to PQ and the wet weight was obtained. The lung tissues were then placed in an oven at $80^{\circ} \mathrm{C}$ for $48 \mathrm{~h}$ and weighed again to obtain the dry weight. Finally, the lung wet/dry (W/D) weight ratio was assessed to assess the severity of pulmonary edema.

$H \& E$ staining and scoring of lung injuries. The middle lobe of the right lung was collected, placed in $4 \%$ paraformaldehyde, and fixed at $4^{\circ} \mathrm{C}$ for at least $48 \mathrm{~h}$. Lung tissues were further subjected to concentration gradient dehydration and wax dipping; the tissues were then embedded in paraffin, cut into $3.5-\mu \mathrm{m}$-thick slices. The sections were deparaffinized with xylene, hydrated, then incubated with hematoxylin for $3 \mathrm{~min}$, washed and stained with eosin for $1 \mathrm{~min}$ at room temperature. Subsequently, the sections were dehydrated with gradient ethanol for $20 \mathrm{~min}$, immersed in xylene to be transparent for 2 min, washed and finally sealed with a neutral gel. After sealing the slides with neutral gum, pathological changes were observed using a microscope (Nikon Corporation) and recorded using NIS-Element software (version 4.6). Pulmonary injury was then scored according to previously described methods (23). Briefly, the following 5-level scale was applied to independently score the pathological changes (alveolar congestion, hemorrhage, and wall thickening, coupled with neutrophil infiltration or aggregation in the alveolar space or blood vessel wall and transparent membrane formation) as follows: $0,1,2,3$ and 4 points represented no or minor, mild, moderate, severe and extremely severe lesions, respectively. Finally, the severity of pulmonary injury was evaluated by the sum of the scores of each pathological feature.

Measurement of superoxide dismutase (SOD) content in lung tissues. The lung tissues were weighed accurately; the homogenates $[10 \%(\mathrm{w} / \mathrm{v})]$ were resuspended in normal saline and centrifuged at 2,500-3,000 $\mathrm{x}$ g at $4^{\circ} \mathrm{C}$ for $10 \mathrm{~min}$. Following instructions provided with the SOD kit (Nanjing Jiancheng Bioengineering Institute), the supernatants were added to a 96-well plate, mixed, and incubated at $37^{\circ} \mathrm{C}$ for $20 \mathrm{~min}$. The absorbance of the sample was measured at $450 \mathrm{~nm}$ using a microplate reader (Elx800, BioTek Instruments, Inc.).

Detection of malondialdehyde (MDA) content in lung tissues. The thiobarbituric acid (TBA) method was used to determine the MDA levels in lung tissue by measuring the absorbance value at $532 \mathrm{~nm}$ using a microplate reader (Elx800, BioTek Instruments, Inc.), according to the instructions provided with the MDA kit (Nanjing Jiancheng Bioengineering Institute).

Cells and cell culture. RAW264.7 cells were obtained from the Cell Bank Type Culture Collection of the Chinese Academy of Sciences. RAW264.7 mouse macrophages were cultured in high-glucose Dulbecco's modified Eagle's medium (DMEM), containing 10\% fetal bovine serum (FBS) and incubated at $37^{\circ} \mathrm{C}$ with $5 \% \mathrm{CO}_{2}$ in a humidified atmosphere. The culture medium was changed daily.

Cell viability assay. Various concentrations of $\mathrm{PQ}(10,50,100$, $250,500$ and $1,000 \mu \mathrm{m})$ or LXA4 $(0,1,10,25,50,100,500$ and $1,000 \mathrm{~nm}$ ) were applied to determine the appropriate PQ and LXA4 experimental concentrations. The effects of PQ or LXA4 on cell viability were assessed using a Cell Counting kit-8 (CCK-8) kit (Dojindo Laboratories, Inc.).

Cell transfection. The GV417 vector-Toll-like receptor 4 (TLR4) plasmid (Shanghai GeneChem Co., Ltd.) was transfected into the cells to achieve the overexpression of TLR4. Specifically, when cells grew to a density of 70-90\%, Lipofectamine 3000 reagent (Thermo Fisher Scientific, Inc.) and the TLR4 overexpression plasmid at the concentration of $10 \mu \mathrm{g} / \mathrm{ml}$ were applied for transfection according to the manufacturer's instructions. Following $24 \mathrm{~h}$ of transfection, the expression level of TLR4 was evaluated by western bot analysis and reverse transcription-quantitative polymerase chain reaction (RT-qPCR).

Establishment of cell models. After selecting the optimal concentration of PQ or LXA4, the RAW264.7 mouse macrophages were divided into 6 groups as follows: i) The 
control (CON) group, cultured in normal medium; ii) PQ poisoning (PQ) group, exposed to medium containing PQ $(100 \mu \mathrm{m})$ for $24 \mathrm{~h}$; iii) PQ poisoning + LXA4 (PQ + LXA4) group, at the point of 1 -h exposure to PQ $(100 \mu \mathrm{m})$, the cells were treated with $10 \mu \mathrm{l}$ LXA4 $(100 \mathrm{~nm})$ for a further $24 \mathrm{~h}$; iv) PQ poisoning + LXA4 + GV417-TLR4 plasmid (PQ + LXA4 + TLR4) group, after 24 h of TLR4-plasmid transfection, the cells were treated as described above for the PQ + LXA4 group; v) PQ poisoning + LXA4 + empty plasmid (PQ + LXA4 + mock) group, after $24 \mathrm{~h}$ of empty vector-plasmid transfection, the cells were then treated as described above for the PQ + LXA4 group; and vi) The LXA4 group, cells were only treated with $10 \mu \mathrm{l} \mathrm{LXA} 4(100 \mathrm{~nm})$ for $24 \mathrm{~h}$. Following $24 \mathrm{~h}$ of treatment, the cells and culture supernatants were collected for use in subsequent experiments.

Reactive oxygen species (ROS) assays. After removing the cell culture solution, DCFH-DA was added, which was diluted in serum-free medium to each plate well, followed by incubation in an incubator at $37^{\circ} \mathrm{C}$ for $30 \mathrm{~min}$. The cells were then washed 3 times with serum-free medium. Images were obtained under a fluorescence microscope (Nikon Corporation).

Determination of pro-inflammatory mediators. Commercially available ELISA kits (EK0526, EK0527, EK0390 and EK0394, Wuhan Boster Biological Technology, Ltd.) were used to measure the tumor necrosis factor (TNF)- $\alpha$ and interleukin (IL)-1 $\beta$ levels in BALF and cell supernatants. The TNF- $\alpha$ and IL-1 $\beta$ levels were calculated according to the standard curve established by the concentration range.

$R T$ - $q P C R$. Total RNA was extracted from the lung tissue and RAW264.7 macrophages using RNAiso Plus reagent (9108, Takara Bio, Inc.). Complementary DNA (cDNA) was synthesized using the PrimeScript RT (with gDNA eraser) kit (RR047A, Takara Bio, Inc.). Subsequently, qPCR was performed using the TB Green Premix Ex Taq ${ }^{\mathrm{TM}}$ II (Tli RNaseH Plus) kit (RR820A, Takara Bio, Inc.) on the Roche Light Cycler 480 system. We carry out the thermocycling according to the following procedure: Initial denaturation at $95^{\circ} \mathrm{C}$ for $30 \mathrm{sec}$, followed by 40 cycles of $5 \mathrm{sec}$ denaturation at $95^{\circ} \mathrm{C}$, annealing and extension at $60^{\circ} \mathrm{C}$ for $20 \mathrm{sec} . \beta$-actin was used as an internal control, and the $2^{-\Delta \Delta \mathrm{Cq}}$ formula was used to calculate relative gene expression. The sequences of the primers used in the present study are listed in Table I.

Western blot analysis. RIPA lysis buffer mixed with protease inhibitor (PMSF) and phosphatase inhibitor (PI) was prepared to extract total protein from the lung tissues or RAW264.7 following exposure to PQ. Nuclear and cytoplasmic protein extraction kits (P0028, Beyotime Institute of Biotechnology) were used to isolate nuclear and cytoplasmic proteins. The concentration of the extracted protein was measured using a BCA assay kit (P0010, Beyotime Institute of Biotechnology), and a total of $50 \mu \mathrm{g}$ protein sample was loaded onto each well of a $10 \%$ sodium dodecyl sulfate polyacrylamide gel for electrophoresis. Following electrophoresis, the proteins were transferred onto a polyvinylidene fluoride (PVDF) membrane followed by incubation in 5\% skim milk or 5\% BSA blocking solution on a rotary shaker at room temperature for $2 \mathrm{~h}$.
Table I. Primer sequences of the genes detected by RT-qPCR.

Gene Primer sequences $\left(5^{\prime}-3{ }^{\prime}\right)$

Rat TLR4

F: ACTTTATCCAGAGCCGTTGGTGTATC R: TCAAGGACAATGAAGATGATGCCAGAG

Rat MyD88 F: CGGCAACTAGAACAGACAGACTATCG R: TCGTCAGAAACAACCACCACCATG

Rat TNF- $\alpha \quad F$ : CCACGCTCTTCTGTCTACTGAACTTC R: TGGGCTACGGGCTTGTCACTC

Rat IL-1 $\quad$ F: AATCTCACAGCAGCATCTCGACAAG R: TCCACGGGCAAGACATAGGTAGC

Rat TRAM F: TCGCAAGAAGAGCAACAAGAACCC R: AGAGCATCGCCAGACAGGAGAC

Rat TRIF F: GTCGCGGAAGTGTGACTAGAAGAG

R: TGCAGCTACCAGAAACCCTCCTC

Rat $\beta$-actin $\quad F$ : TGTCACCAACTGGGACGATA

R: GGGGTGTTGAAGGTCTCAAA

Mouse TLR4 F: GCCATCATTATGAGTGCCAATT

R: AGGGATAAGAACGCTGAGAATT

Mouse F: CGGAACTTTTCGATGCCTTTAT

MyD88 R: CACACACAACTTAAGCCGATAG

Mouse F: ATGTCTCAGCCTCTTCTCATTC

TNF- $\alpha$ R: GCTTGTCACTCGAATTTTGAGA

Mouse IL-1 $\beta$ F: TCGCAGCAGCACATCAACAAGAG

R: AGGTCCACGGGAAAGACACAGG

Mouse F: GACAGTGTGGATGCCGATCAAGAC

TRAM R: TGGTCCTGCTCTCCTGTTGGTG

Mouse TRIF F: ACGATCCTGCTCCTGACTGCTAG

R: TCCTGCCTCCCAGACTGTGTAAG

Mouse Actb F: GTGCTATGTTGCTCTAGACTTCG

R: ATGCCACAGGATTCCATACC

Subsequently, the membranes with proteins were incubated on a shaker at $4^{\circ} \mathrm{C}$ overnight with the following primary antibodies: TLR4 (1:1,000, \#AF7017), myeloid differentiation primary response 88 (MyD88; 1:1,000, \#AF5195), phosphoinositide 3-kinase (PI3K; 1:1,000, \#AF6241), phosphorylated (p-)PI3K (1:1,000, \#AF3242) (all from Affinity Biosciences); AKT (1:1,000,\#4691), p-AKT (1:2,000,\#4060), $\mathrm{I} \kappa \mathrm{B} \alpha(1: 1,000, \# 4814)$ and $\mathrm{p}-\mathrm{I} \kappa \mathrm{B} \alpha(1: 1,000, \# 2859)$ (all from Cell Signaling Technology, Inc.); nuclear factor (NF)- $\kappa$ B p65 (1:500, ab16502, Abcam); TATA box binding protein (TBP; $1: 2,000, \# 22006-1-A P)$ and $\beta$-actin (1:2000, \#20536-1-AP) (both from ProteinTech Group, Inc.). The following day, the membranes were washed using Tris-buffered saline with Tween-20 (TBST; Sigma-Aldrich; Merck KGaA) 3 times and incubated with the HRP-conjugated secondary antibody (\#ZB-5301, ZSJQB Co., Ltd.) for $1 \mathrm{~h}$ at room temperature. After washing a further 3 times, enhanced chemiluminescence (ECL) (KF003-100, Affinity Biosciences) solution was applied to visualize the immunoreactive bands, and ImageJ (version 1.8.0) software was used for quantitative image analysis. 
A
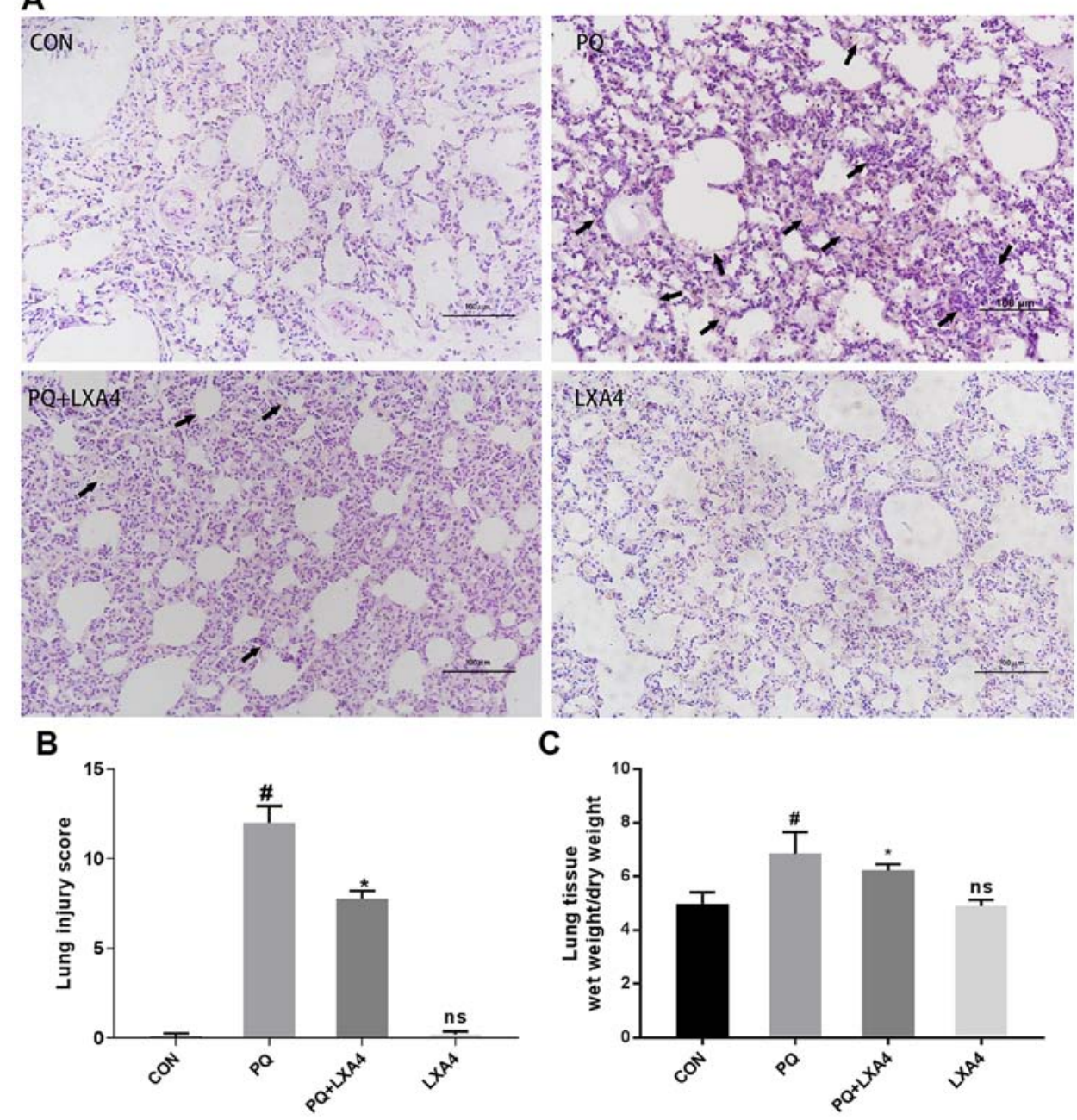

Figure 1.LXA4 alleviates PQ-induced lung damage in rats. (A) Lung tissue damage assessment by H\&E staining, (B) lung injury score evaluation, and (C) lung W/D ratios in each group. Data are presented as the means $\pm \mathrm{SD}(\mathrm{n}=10) .{ }^{*} \mathrm{P}<0.05$ vs. the CON group. ${ }^{*} \mathrm{P}<0.05$ vs. the $\mathrm{PQ}$ group. ns, not significant $(\mathrm{P}>0.05)$ vs. the CON group. LXA4, lipoxin A4; PQ, paraquat.

Statistical analysis. The data are expressed as the means \pm standard deviation (SD). One-way analysis of variance (ANOVA) and Tukey's test was performed to analyze the statistical differences between each group. A P-value $<0.05$ was considered to indicate a statistically significant difference. All statistical analyses were performed using Graphpad Prism 7.0 software.

\section{Results}

LXA4 alleviates lung histopathological damage induced by $P Q$. As shown in Fig. 1A, histopathological injuries were observed in the PQ group, reflected by alveolar structure destruction, alveolar congestion or bleeding, alveolar cavity collapse, inflammatory cell infiltration and the formation of transparent film in part of the alveolar cavity. In the poisoned group treated with LXA4, the histopathological injuries were all attenuated, together with decreased pulmonary hemorrhage and edema. Significant histopathological differences were not observed between the CON and LXA4 groups. The histological scores of the PQ group were significantly higher than those of the CON group, while the use of LXA4 substantially attenuated the lung injury scores (Fig. 1B).
$L X A 4$ reduces the lung W/D weight ratio in rats exposed to $P Q$. The lung W/D weight ratio was measured in each group of rats to confirm the protective effects of LXA4 on pulmonary edema induced by $\mathrm{PQ}$. As shown in Fig. $1 \mathrm{C}$, the W/D ratio of the PQ-treated rat lungs was higher compared to that of the CON group, but was markedly reduced by LXA4.

LXA4 attenuates the inhibitory effects on the viability of RAW264.7 cells induced by PQ. CCK-8 assay was used to evaluate the optimal concentration of PQ for use in subsequent experiments. The results suggested that the inhibitory effect of PQ on the viability of RAW264.7 cells occurred in a concentration-dependent manner. When the PQ concentration was $\geq 100 \mu \mathrm{M}$, RAW264.7 cell viability was markedly inhibited (Fig. 2A). Therefore, PQ at $100 \mu \mathrm{M}$ was used as the optimum dose in subsequent in vitro experiments. The same method was used to determine the possible toxic effects of LXA4 on RAW264.7 macrophages and the optimal concentration of LXA4 that could reduce PQ cytotoxicity. Notably, in the present study, it was found that treatment with LXA4 alone had no toxic effect on the RAW264.7 cells (Fig. 2B), and when the LXA4 concentration was $100 \mathrm{nM}$, its ability to reduce PQ cytotoxicity was optimal (Fig. 2C). Thus, the concentration of 
A

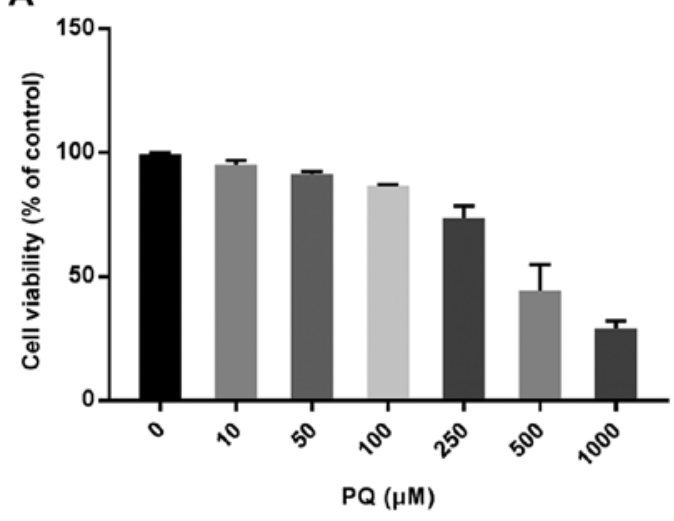

B

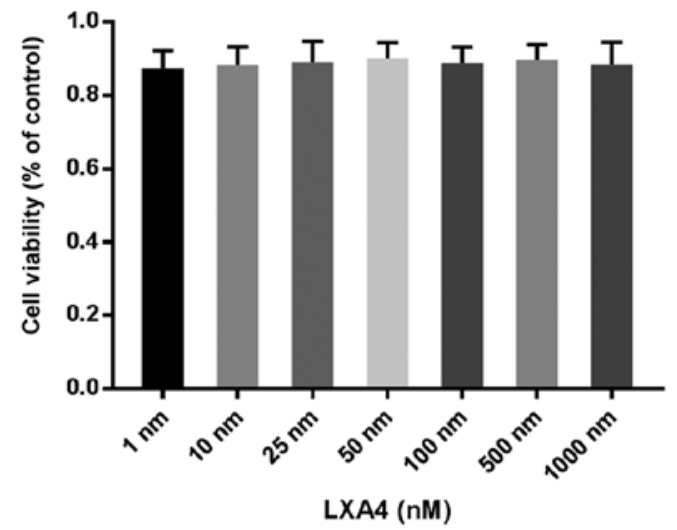

C

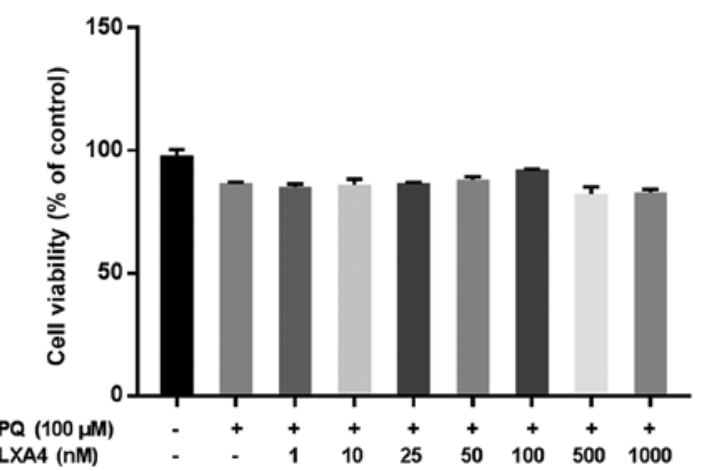

Figure 2. LXA4 facilitates the viability of RAW264.7 cells. (A) RAW264.7 cell viability was suppressed when cells were treated with various PQ concentrations. (B) LXA4 had no toxic on RAW264.7 cells. (C) LXA4 improved RAW264.7 cell viability. Data are presented as the means \pm SD (n=3). LXA4 was added $1 \mathrm{~h}$ prior to PQ treatment. LXA4, lipoxin A4; PQ, paraquat.

$100 \mathrm{nM}$ LXA4 was selected as the optimum dose for use in subsequent studies.

LXA4 reduces oxidative stress in lung tissues and ROS generation in RAW264.7 macrophages. As shown in Fig. 3A and $\mathrm{B}$, lung SOD activity significantly decreased following exposure to $\mathrm{PQ}$, while the MDA content markedly increased; however, LXA4 treatment significantly restored SOD activity and decreased the MDA content. The ROS expression level in the RAW264.7 macrophages increased significantly following exposure to PQ, whereas this decreased upon treatment with LXA4 (Fig. 3C). These results indicated that LXA4 exerted beneficial effects on PQ-induced oxidative damage.

LXA4 inhibits the PQ-induced increase in the levels of pro-inflammatory cytokines. ELISA was used to measure the levels of pro-inflammatory factors in BALF and RAW264.7 cell supernatants. The results revealed that PQ markedly increased TNF- $\alpha$ and IL-1 $\beta$ secretion, while LXA4 treatment significantly reduced these levels (Figs. 4A and B). Simultaneously, the mRNA expression of inflammatory factors was determined to further evaluate the effects of LXA4 on PQ. The results revealed that the expression levels of TNF- $\alpha$ and IL-1 $\beta$ were significantly upregulated in lung tissues and RAW264.7 cells following PQ exposure, whereas LXA4 administration reversed these effects (Fig. 4C and D).

LXA4 inhibits PQ-induced TLR4 and MyD88 overexpression. In order to explore the possible immune mechanisms responsible for the protective effects of LXA4 against PQ-induced ALI, RT-qPCR and western blot analysis were performed to detect the mRNA and protein levels of key molecules participating in the TLR4 signaling pathway in rat lung tissues and RAW264.7 cells. As shown in Fig. 5, LXA4 administration significantly reduced the elevated levels of TLR4 and MyD88 induced by PQ. However, as for the other pathway mediated by TLR4, also known as the Myd88-independent pathway, there was no significant difference in the mRNA expression levels of TRAM and TRIF between the PQ and PQ + LXA4 group. These results indicated that PQ poisoning activated the TLR4 signaling pathway and that the protective effects of LXA4 were mediated by the MyD88-dependent pathway of TLR4.

LXA4 suppresses the activation of the $N F-\kappa B$ pathway. The activation of TLR4/MyD88 stimulates $\mathrm{I} \kappa \mathrm{B} \alpha$ phosphorylation and the nuclear transfer of $\mathrm{NF}-\kappa \mathrm{B}$ p65, thereby activating the release of pro-inflammatory cytokines $(24,25)$. Therefore, the present study examined the effects of PQ and LXA4 on the $N F-\kappa B$ signaling pathway to elucidate the mechanisms through which LXA4 inhibits PQ-induced inflammation. The results from the in vitro and in vivo experiments indicated that PQ significantly promoted the process of $\mathrm{I} \kappa \mathrm{B} \alpha$ degradation to $\mathrm{p}-\mathrm{I} \kappa \mathrm{B} \alpha$, leading to an increase in the transfer of $\mathrm{NF}-\kappa \mathrm{B}$ p65 to the nucleus. However, in the PQ + LXA4 group, the PQ-induced overactivation of the NF- $\kappa \mathrm{B}$ signaling pathway was markedly attenuated (Fig. 6), indicating that inflammatory responses could be inhibited by LXA4 via the suppression of the NF- $\kappa \mathrm{B}$ pathway. 
A

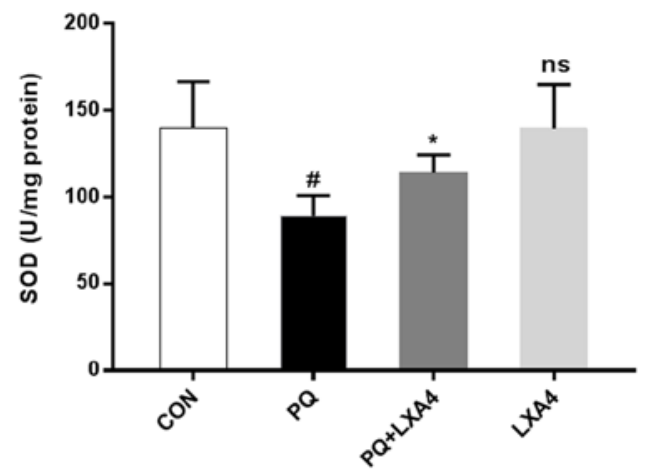

C
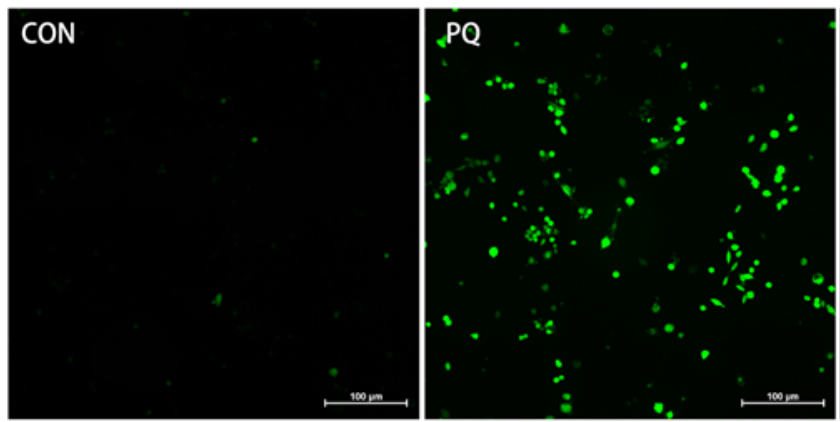

B
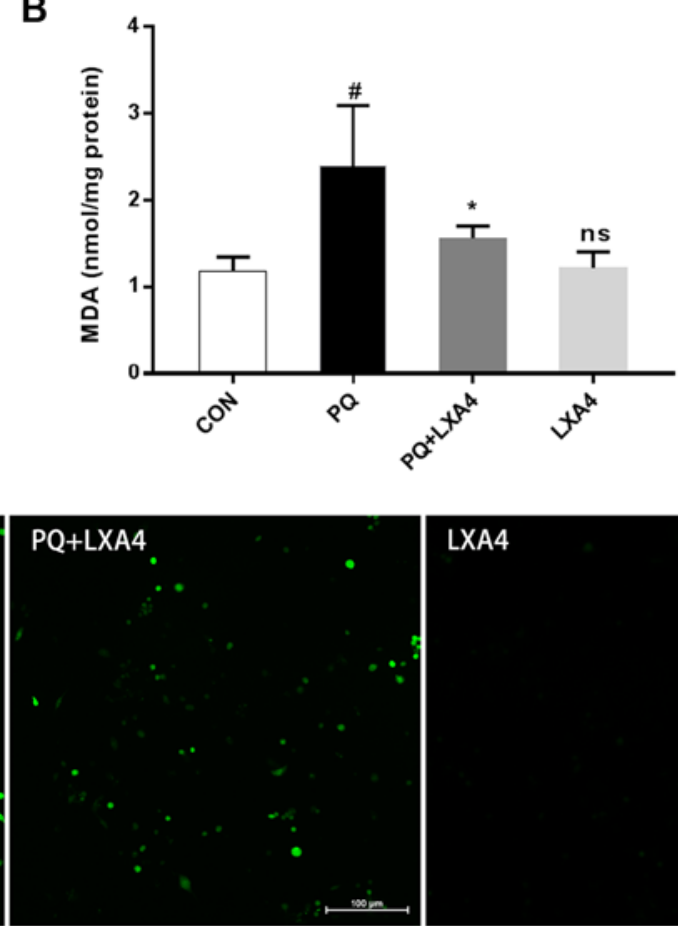

LXA4

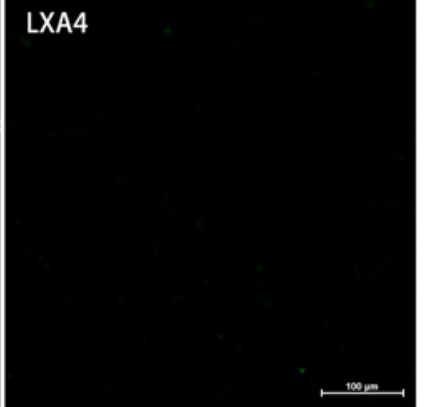

Figure 3. LXA4 attenuates the degree of oxidative stress. (A) SOD activity in rat lung tissues. (B) MDA content in rat lung tissues. (C) The fluorescent probe, DCFH-DA, was used to assess the ROS levels in RAW264.7 cells. Data are presented as the means $\pm \mathrm{SD}$ ( $\mathrm{n}=10$ in vivo). ${ }^{\#} \mathrm{P}<0.05$ vs. the CON group. ${ }^{\#} \mathrm{P}<0.05$ vs. the CON group. ${ }^{*} \mathrm{P}<0.05$ vs. the PQ group. ns, not significant $(\mathrm{P}>0.05)$ vs. the CON group. LXA4, lipoxin A4; PQ, paraquat.

A

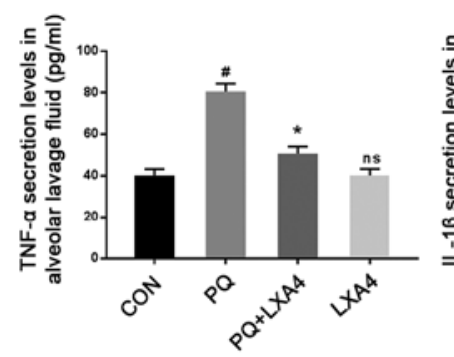

C

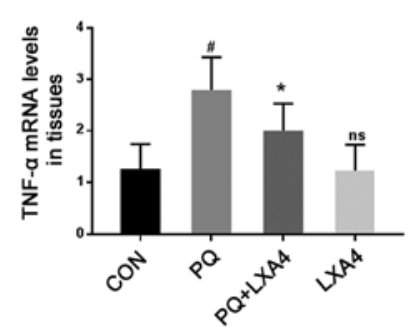

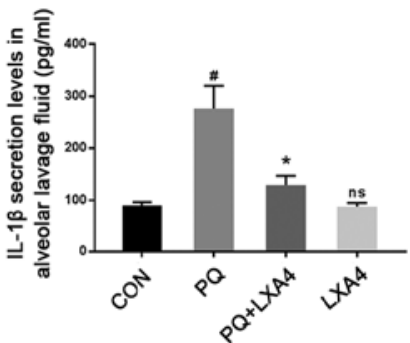

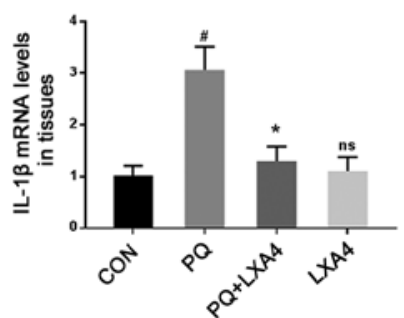

B
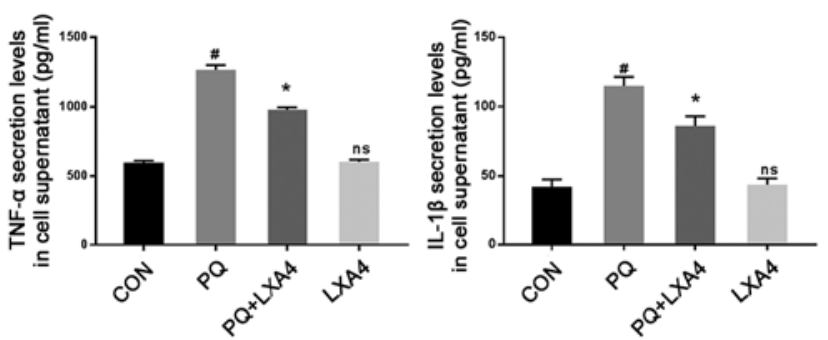

D

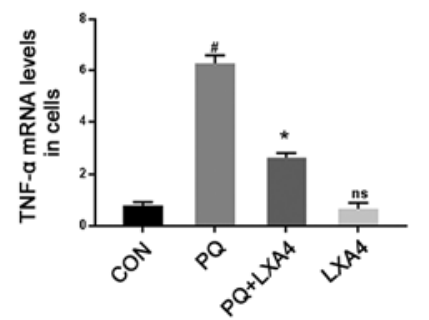

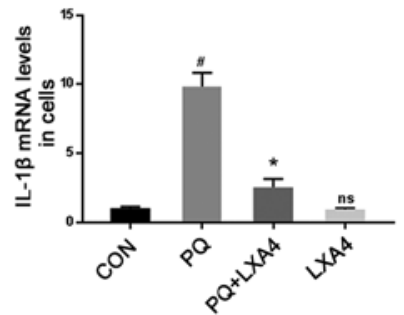

Figure 4. LXA4 inhibits the expression levels of TNF- $\alpha$ and IL-1 $\beta$. The expression levels of TNF- $\alpha$ and IL-1 $\beta$ in (A) BALF and (B) the culture supernatants of RAW264.7 cells. mRNA expression levels of TNF- $\alpha$ and IL-1 $\beta$ in (C) rat lungs and (D) RAW264.7 cells. Data are presented as the means \pm SD ( $\mathrm{n}=10$ in vivo, $\mathrm{n}=6$ in vitro). ${ }^{"} \mathrm{P}<0.05$ vs. the $\mathrm{CON}$ group. ${ }^{*} \mathrm{P}<0.05$ vs. the $\mathrm{PQ}$ group. $\mathrm{ns}$, not significant $(\mathrm{P}>0.05)$ vs. the CON group. LXA4, lipoxin $\mathrm{A} 4 ; \mathrm{PQ}$, paraquat.

LXA4 suppresses the activation of the PI3K/AKT pathway. Previous studies (26-28) have demonstrated that activated TLR4/MyD88 can also activate the PI3K/AKT signaling pathway and induce inflammatory responses. Therefore, the present study investigated whether the PI3K/AKT signaling pathway was also involved in the anti-inflammatory effects of LXA4 to further clarify the cellular mechanisms responsible for the protective effects of LXA4 on PQ-induced ALI. As shown in Fig. 7, PQ stimulation significantly upregulated the phosphorylation of PI3K and AKT, both of which were suppressed by LXA4 intervention. These results indicated that the activation fo the PI3K/AKT pathway induced by PQ was inhibited by LXA4.

Mitigated protection of LXA4 following the enforced overexpression of TLR4. In order to determine whether 

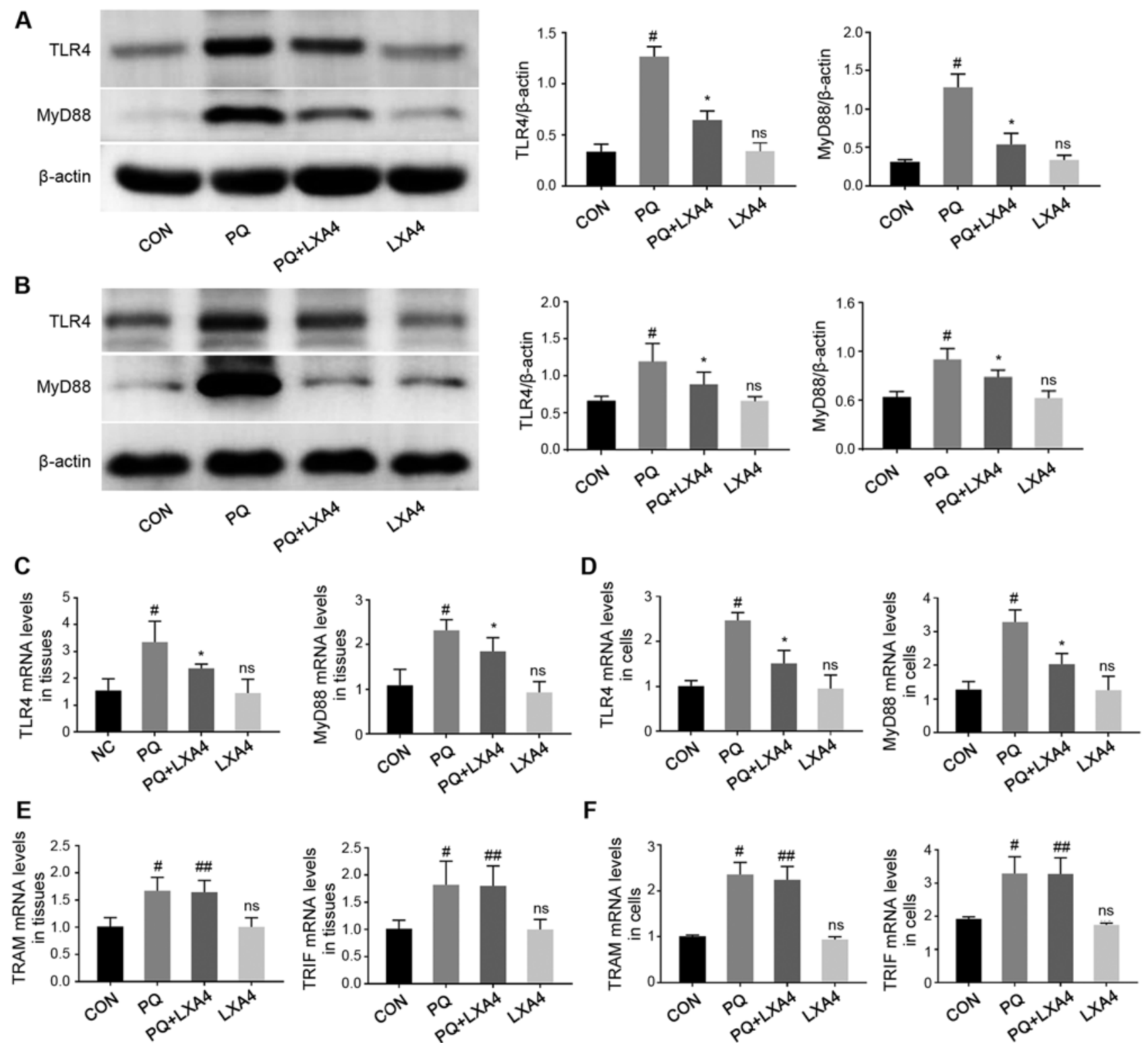

$\mathbf{F}$
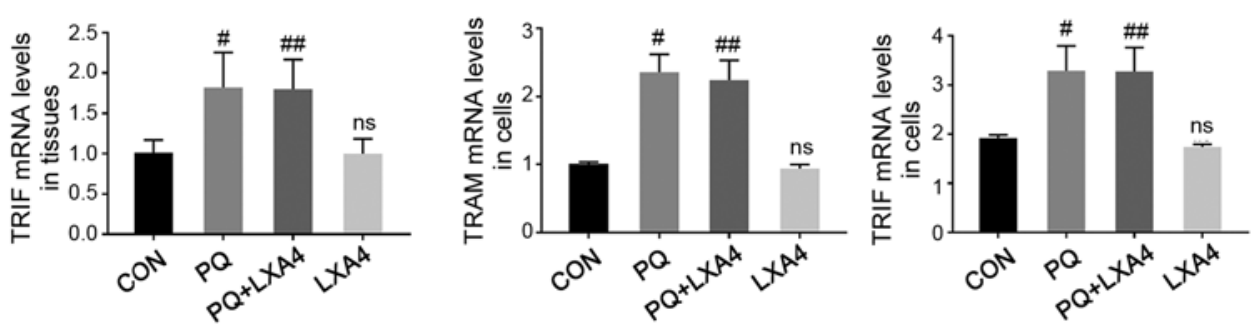

Figure 5. LXA4 inhibits TLR4 and MyD88 overexpression induced by PQ. Western blot analysis was performed to assess the protein levels of TLR4 and MyD88 in (A) rat lung tissues and (B) RAW264.7 cells. RT-qPCR analyses were performed to determine the gene levels of TLR4, MyD88, TRAM and TRIF in $(\mathrm{C}$ and $\mathrm{E})$ rat lungs and $(\mathrm{D}$ and $\mathrm{F}) \mathrm{RAW} 264.7$ cells. Data are presented as the means $\pm \mathrm{SD}\left(\mathrm{n}=10\right.$ in vivo, $\mathrm{n}=6$ in vitro). ${ }^{\#} \mathrm{P}<0.05$ vs. the $\mathrm{CON}$ group. ${ }^{*} \mathrm{P}<0.05$ vs. the PQ group. ns, not significant $(\mathrm{P}>0.05)$ vs. the CON group. ${ }^{\# \#} \mathrm{P}>0.05$ vs. the PQ group. LXA4, lipoxin $\mathrm{A} 4$; $\mathrm{PQ}$, paraquat.

the protective effects of LXA4 PQ poisoning are mediated by the suppression of TLR4, a GV417-TLR4 plasmid was constructed, and rescue experiments were conducted in vitro. As shown in Fig. 8A, the protein and mRNA expression of TLR4 was significantly higher in the TLR4 overexpression group than that in the CON and mock-transfection groups. The results also revealed that the enforced overexpression of TLR4 reversed the protective effects of LXA4 against PQ-induced ROS production and pro-inflammatory cytokine secretion (Fig. 8B and C). In addition, the Myd88, NF- $\kappa \mathrm{B}$ and PI3K/AKT pathways inhibited by LXA4 were all re-activated following transfection with TLR4 overexpression plasmid (Fig. 9). Therefore, these findings verified that LXA4 exerted protective effects against PQ poisoning through the suppression of TLR4 and its downstream MyD88-mediated $\mathrm{NF}-\kappa \mathrm{B} / \mathrm{PI} 3 \mathrm{~K} / \mathrm{AKT}$ signaling pathway.

\section{Discussion}

PQ is a highly toxic chemical herbicide and one of the most common drugs that causes acute poisoning and severe damage to multiple organs. Previous studies have proven that the lungs are a specific target organ for pathological damages caused by PQ (29). After PQ enters the body, it rapidly becomes transported into the lung tissue through the polyamine transportation system, resulting in ALI or even ARDS in the early stage of poisoning. Currently, the mechanism of PQ-induced ALI is mainly attributed to oxidative stress and inflammatory response. In the present study, LXA4 was confirmed for the first time to effectively attenuate lung damage caused by $\mathrm{PQ}$, and this protective effect was reflected by the inhibition of pulmonary edema, inflammatory responses and oxidative stress. Simultaneously, it was found that this beneficial 
A

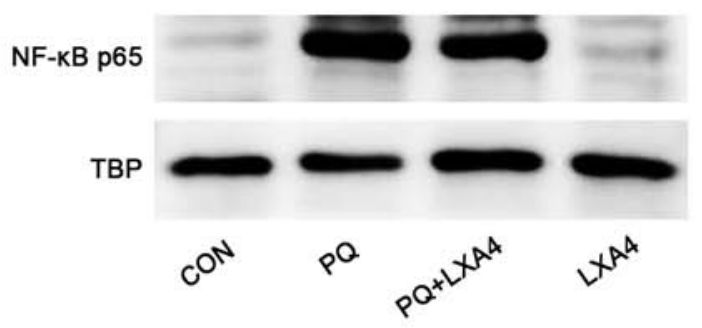

B

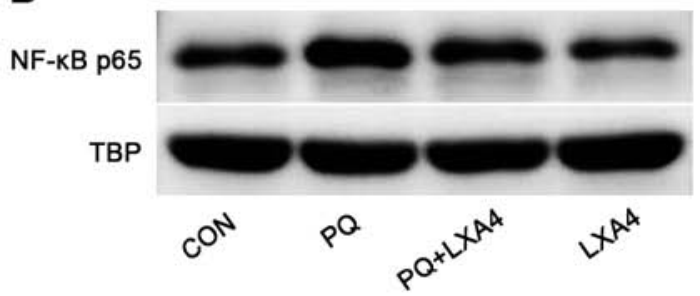

C

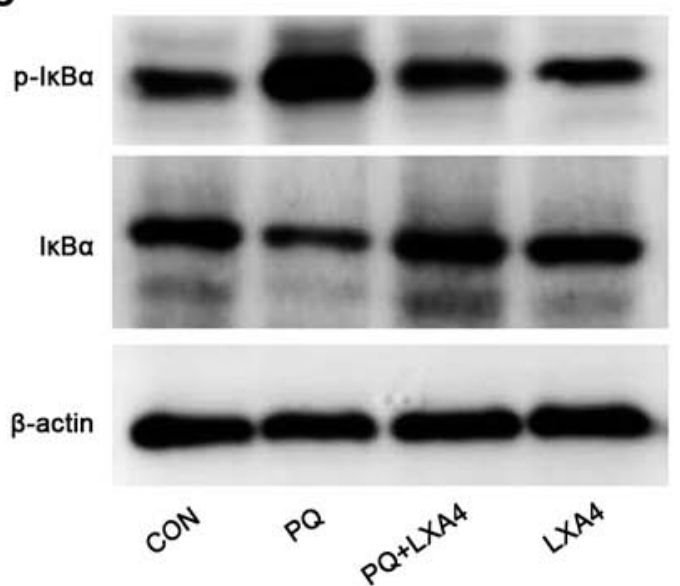

D

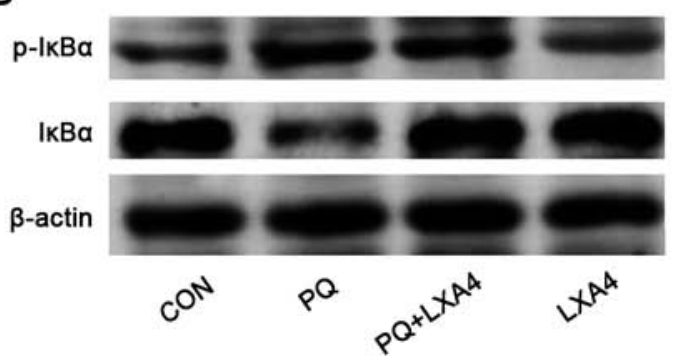

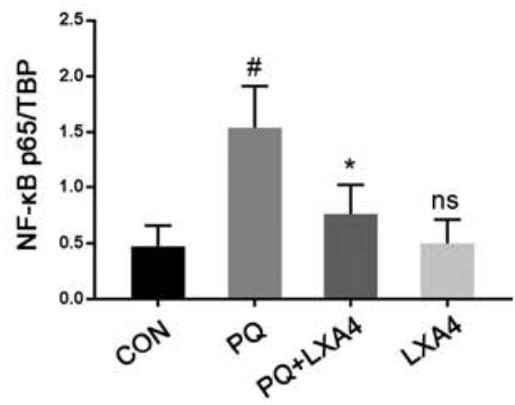
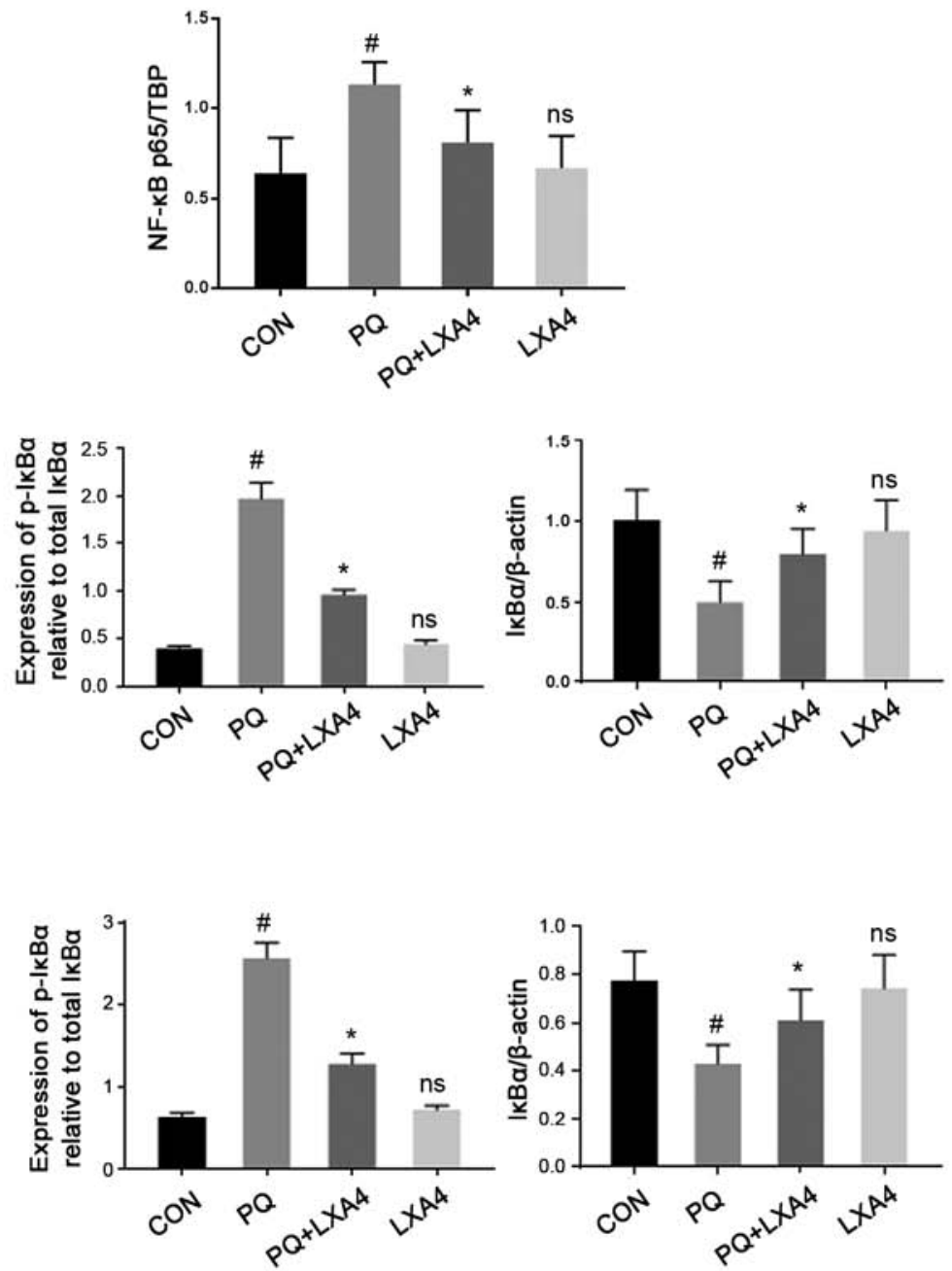

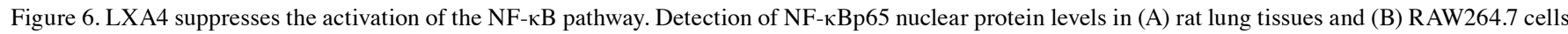
by western blot analysis. Observation of total $\mathrm{I} \kappa \mathrm{B} \alpha$ and $\mathrm{p}-\mathrm{I} \kappa \mathrm{B} \alpha$ protein in $(\mathrm{C})$ rat lungs and (D) RAW264.7 cells. Data are presented as the means $\pm \mathrm{SD}$ ( $\mathrm{n}=10$ in vivo, $\mathrm{n}=6$ in vitro). ${ }^{\#} \mathrm{P}<0.05$ vs. the $\mathrm{CON}$ group. ${ }^{*} \mathrm{P}<0.05$ vs. the $\mathrm{PQ}$ group. ns, not significant $(\mathrm{P}>0.05)$ vs. the CON group. LXA4, lipoxin $\mathrm{A} 4$; $\mathrm{PQ}$, paraquat.

effect of LXA4 was associated with the suppression of the TLR4/MyD88-mediated NF- $\mathrm{B} / \mathrm{PI} 3 \mathrm{~K} / \mathrm{AKT}$ signaling pathway.

LXA4 is an anti-inflammatory mediator produced by the body itself during the late stage of acute inflammation. Previous studies have confirmed its protective effects against inflammation in some diseases, such as neuromyelitis optical spectrum disorders (30), obesity-induced adipose inflammation (31) and cerebrovascular endothelial dysfunction (32). The results from the present study suggested that PQ caused typical ALI and induced severe inflammatory responses in lung tissues and RAW264.7 macrophages, mainly manifested by the destruction of alveolar integrity, inflammatory cell infiltration, alveolar wall thickening, pulmonary edema and upregulated levels of related pro-inflammatory cytokines. Following LXA4 intervention, these injuries and overactive inflammatory responses were alleviated.

SOD activity reflects the ability of the body to scavenge oxygen-free radicals and is often used as a marker of the antioxidant status of the body (33), while MDA levels reflect the degree of oxidative stress damage to the body (34). PQ poisoning not only induces excessive ROS production and impairs ROS clearance, leading to oxidative stress reactions, but also interrupts the balance between SOD and MDA, which is important for the antioxidant capacity of the body. However, 
A
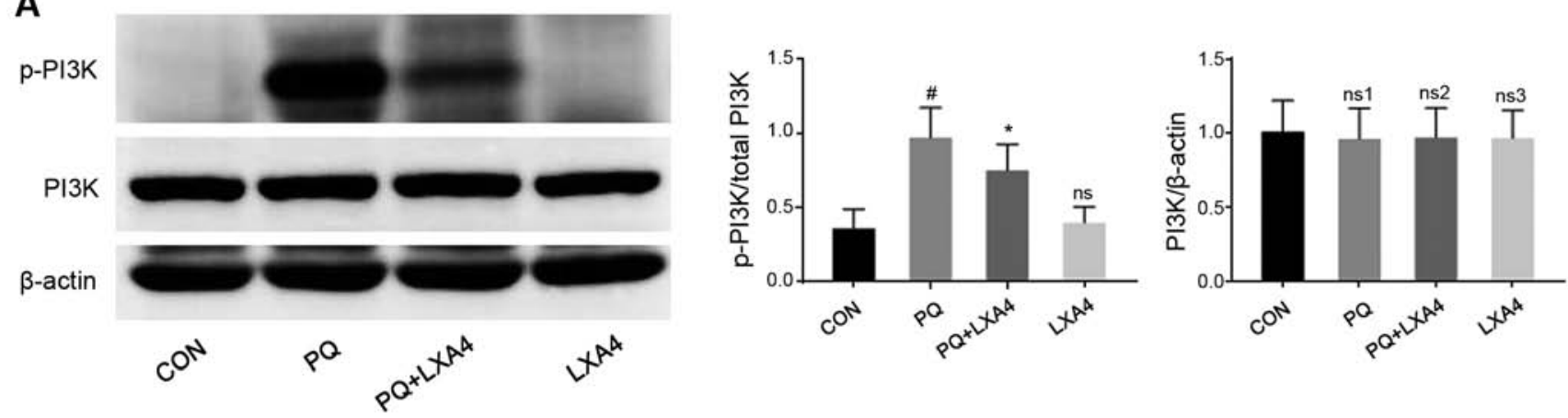

B
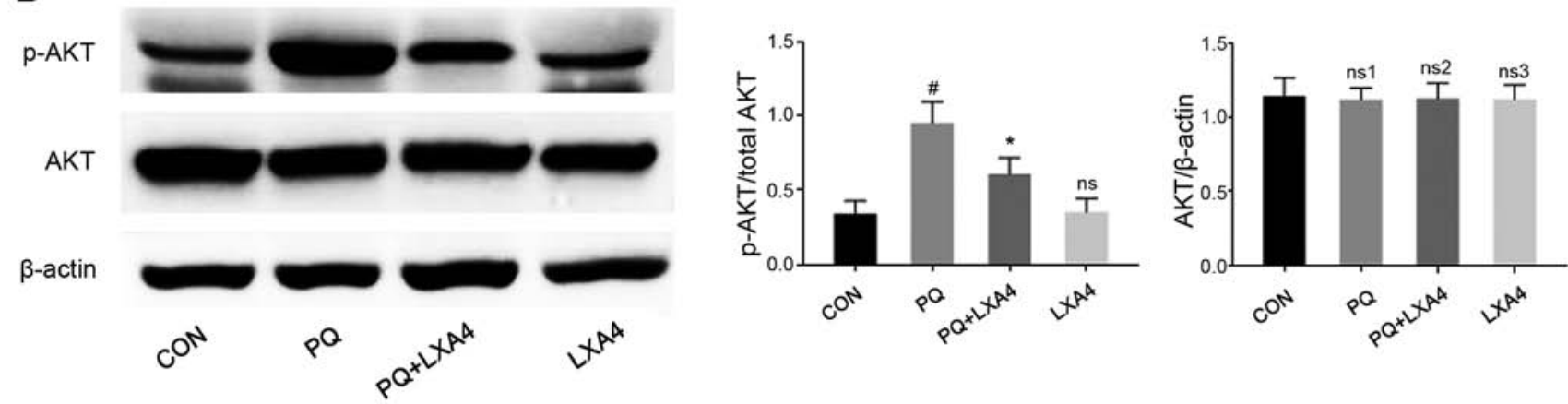

C
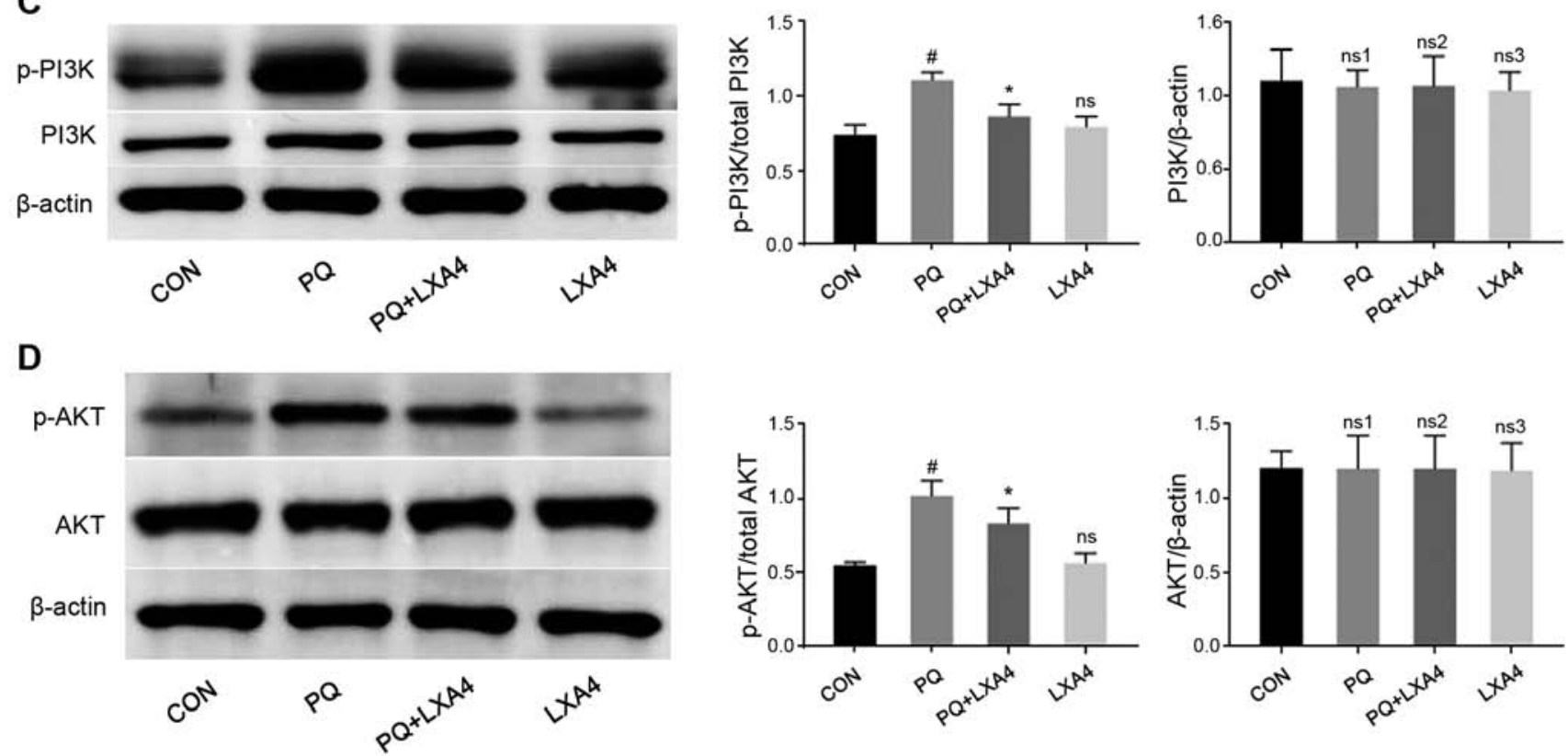

Figure 7. LXA4 suppresses the activation of the PI3K/AKT pathway induced by PQ. Western blot analysis was performed to detect the protein levels of total-PI3K, p-PI3K, total AKT and p-AKT in (A and B) rat lung tissues and ( $\mathrm{C}$ and D) RAW264.7 cells. Data are presented as the means \pm SD (n=10 in vivo, $\mathrm{n}=6$ in vitro). ${ }^{*} \mathrm{P}<0.05$ vs. the $\mathrm{CON}$ group. ${ }^{*} \mathrm{P}<0.05$ vs. the $\mathrm{PQ}$ group. ns, $\mathrm{ns} 1, \mathrm{~ns} 3$, not significant $(\mathrm{P}>0.05)$ vs. the CON group; ns 2 , not significant $(\mathrm{P}>0.05)$ vs. the PQ group. LXA4, lipoxin A4; PQ, paraquat.

in the present study, following LXA4 treatment, these conditions improved, as LXA4 restored the balance between SOD and MDA, thus enhancing the ability of the body to resist oxidative stress damage.

TLRs serve as significant pattern recognition receptors in the host defense system and recognize damage-associated molecular patterns (DAMPs) released by non-infectious stimuli $(35,36)$. Due to the ability to recognize PQ-induced DAMP, TLR4 activates a downstream cascade of inflammatory signals through the MyD88-dependent pathway (37-39). Consistent with the study by Shen et al (24), the present study found that TLR4 and MyD88 expression significantly increased following exposure to $\mathrm{PQ}$, and similar trends were observed in the downstream molecules of TLR4 and MyD88 (such as NF- $\kappa$ B p65, TNF- $\alpha$ and IL-1 $\beta$ ). Owing to the beneficial effects of inhibiting the inflammatory factors, TLR4 and MyD88, the present study investigated whether the protective effects of LXA4 were closely related to TLR4 and MyD88 suppression. The results of the present study suggested that LXA4 inhibited the expression levels of TLR4, MyD88, and their downstream inflammatory cytokines, whereas had no effect on TRAM and TRIF, indicating that the anti-inflammatory effects of LXA4 

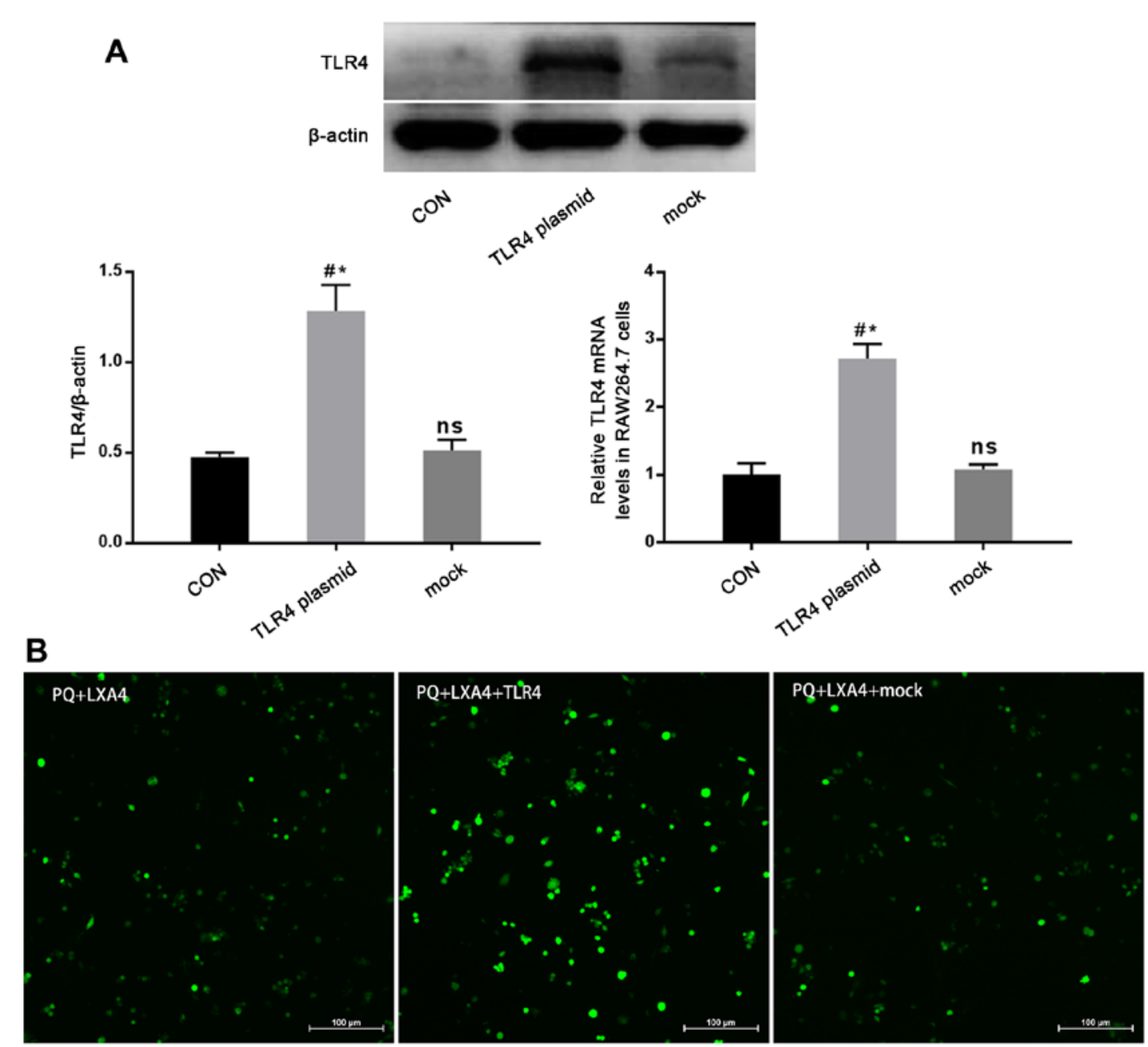

C
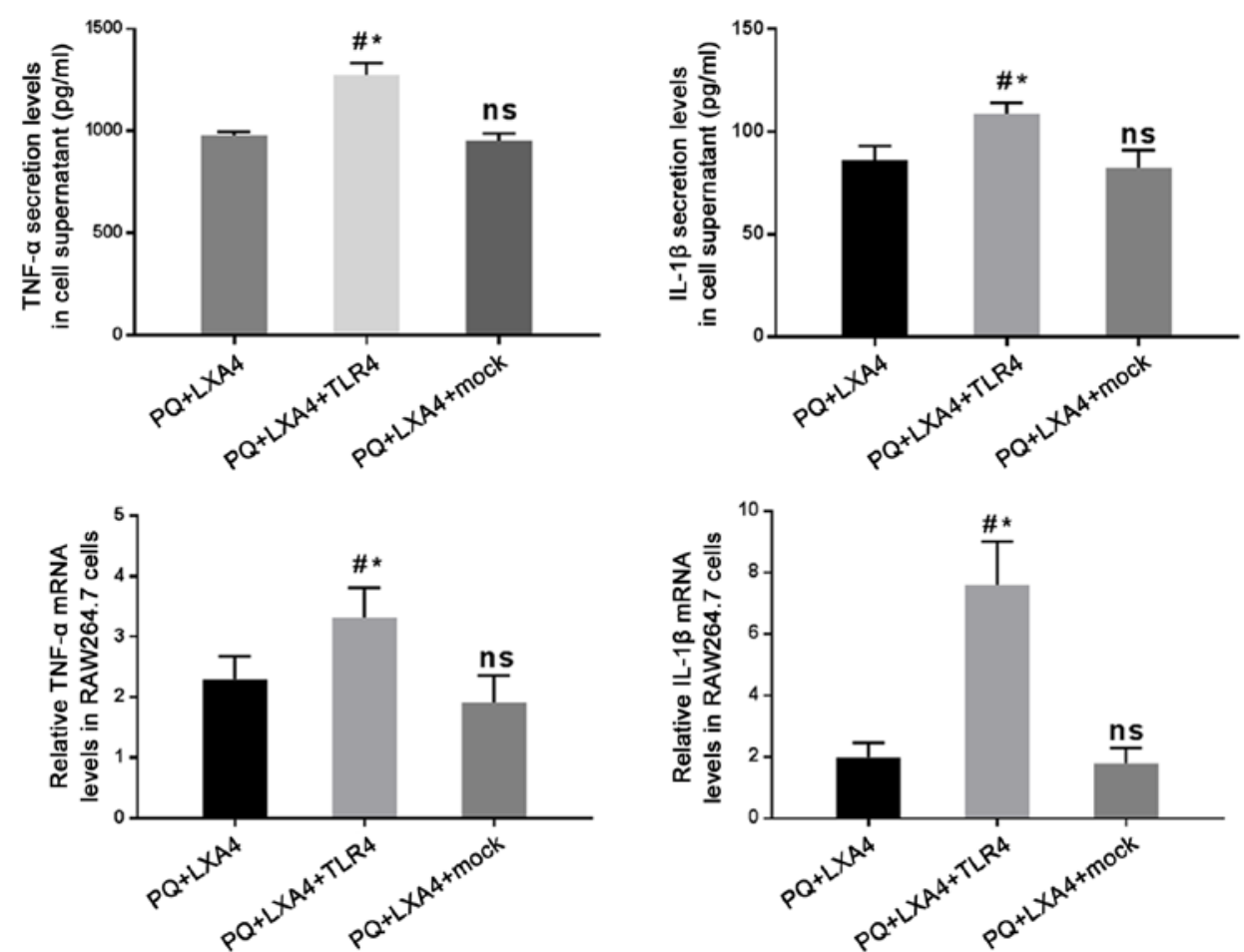

Figure 8. Mitigated protection of LXA4 following the enforced overexpression of TLR4. (A) Western blot analysis and RT-qPCR were performed to determine the expression of TLR4 protein and mRNA in the GV417-TLR4-transfected RAW264.7 cells. Data are presented as the means $\pm \mathrm{SD}(\mathrm{n}=3)$. ${ }^{*} \mathrm{P}<0.05$ vs. the CON group. " $\mathrm{P}<0.05$ vs. the mock group. ns, not significant $(\mathrm{P}>0.05)$ vs. the CON group. (B) The fluorescent probe, DCFH-DA, was used to assess the ROS expression levels in the GV417-TLR4-transfected RAW264.7 cells. (C) The expression levels of TNF- $\alpha$ and IL-1 $\beta$ in the culture supernatants and mRNA expression levels of TNF- $\alpha$ and IL-1 $\beta$ in RAW264.7 cells. Data are presented as the means $\pm \mathrm{SD}(\mathrm{n}=6) .{ }^{*} \mathrm{P}<0.05$ vs. the $\mathrm{PQ}+\mathrm{LXA} 4$ group. ${ }^{*} \mathrm{P}<0.05$ vs. the PQ + LXA4 + mock group. ns, not significant $(\mathrm{P}>0.05)$ vs. the PQ + LXA4 group. LXA4, lipoxin A4; PQ, paraquat; TNF- $\alpha$, tumor necrosis factor $\alpha$; IL-1 $\beta$, interleukin $1 \beta$. 
A

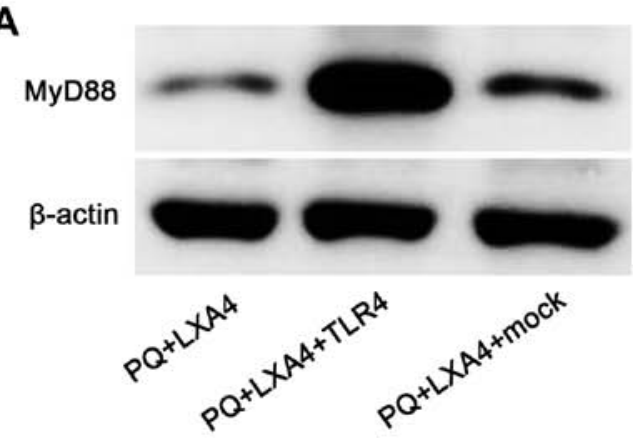

B

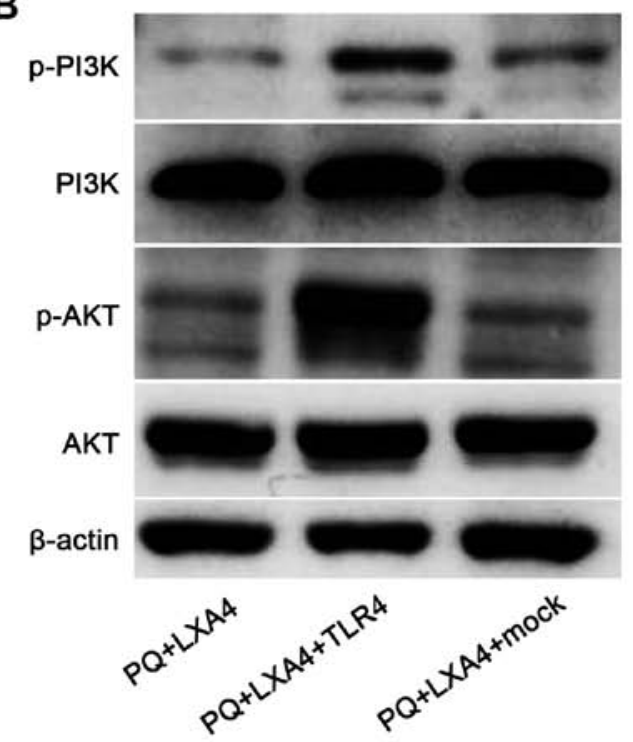

C

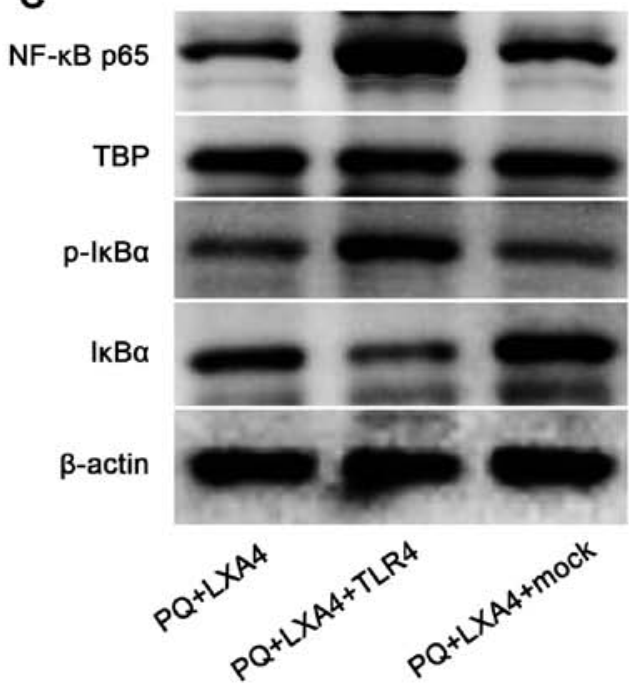

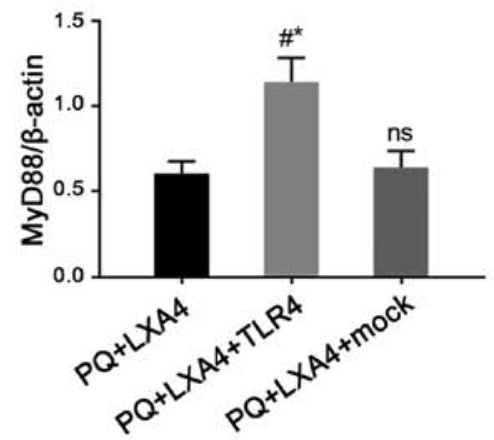
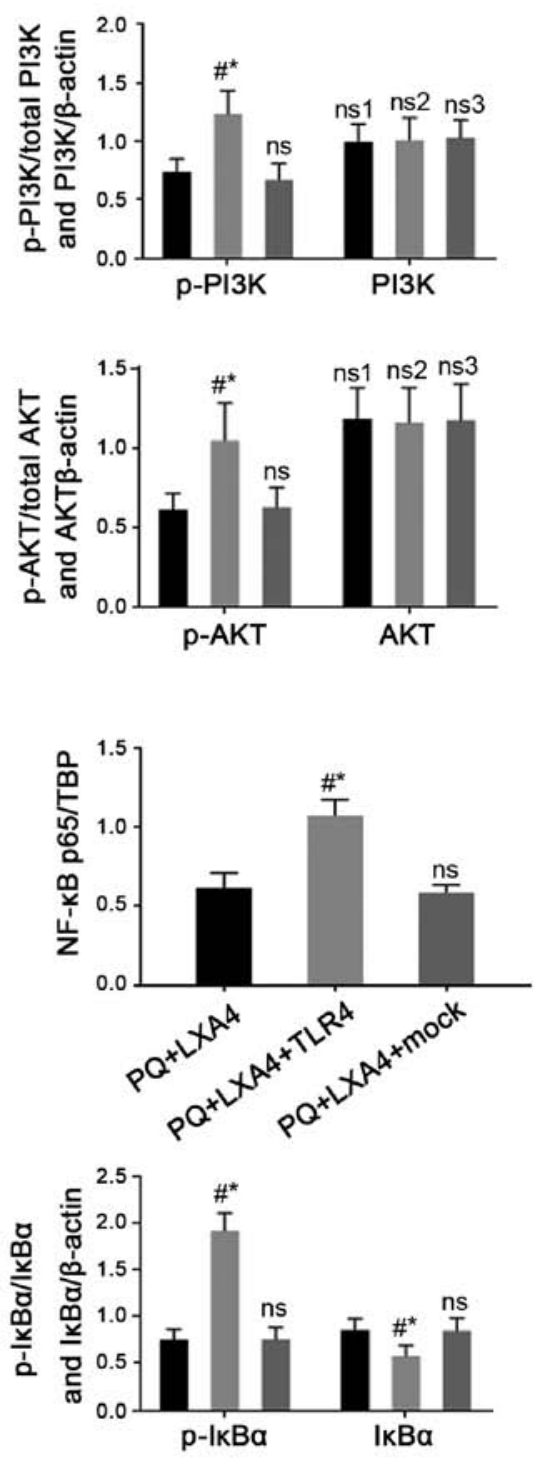

Figure 9. Mitigated protection of LXA4 following the enforced overexpression of TLR4. Detection of (A) MyD88, and (B) total PI3K, p-PI3K, total AKT and

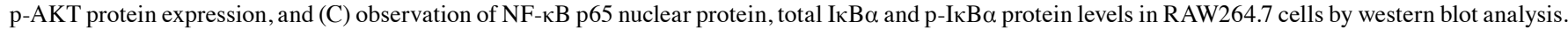
Data are presented as the means $\pm \mathrm{SD}(\mathrm{n}=6) .{ }^{\text {" }} \mathrm{P}<0.05$ vs. the $\mathrm{PQ}+\mathrm{LXA} 4$ group. ${ }^{*} \mathrm{P}<0.05$ vs. the $\mathrm{PQ}+\mathrm{LXA} 4+$ mock group. ns, not significant; ns3, $\mathrm{P}>0.05$ vs. the PQ + LXA4 group; ns1, P>0.05 vs. the PQ + LXA4 + TLR4 group; ns2, P>0.05 vs. the PQ + LXA4 + mock group. LXA4, lipoxin A4; PQ, paraquat.

on PQ may be attributed to the suppression of the TLR4 and MyD88-dependent signaling pathway.

The NF- $\mathrm{KB}$ transcription factor, a typical pro-inflammatory signaling factor, has long been considered a downstream transduction factor of TLR4/MyD88, and this feature is based on its critical effect on promoting the release of pro-inflammatory cytokines (40-42). Ideally, the RelA/p65 and $\mathrm{p} 50$ subunits of NF- $\mathrm{KB}$ constitute heterodimers, which bind to I $\kappa \mathrm{B} \alpha$ (the NF- $\kappa \mathrm{B}$ inhibitor), keeping NF- $\kappa \mathrm{B}$ inactive in the cytoplasm. Stimulated by external injuries, IкB $\alpha$ becomes phosphorylated and degraded, thereby promoting $\mathrm{NF}-\mathrm{\kappa B}$ activation and translocation to the nucleus, where activated NF- $\mathrm{KB}$ promotes the occurrence and development of downstream inflammatory reactions (43-45). The results 
of the present study demonstrated that LXA4 decreased

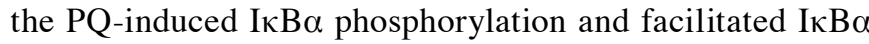
degradation, resulting in the reduced nuclear transfer of NF- $\mathrm{BB}$ p65. Therefore, it was confirmed that the beneficial effects of LXA4 on PQ poisoning were associated with the inhibition of the NF-KB pathway.

The PI3K/AKT pathway, which is closely related to TLR4/MyD88 (46) and reported as a regulator of NF- $\kappa \mathrm{B}$, can enhance the transcriptional activity and nuclear translocation of NF- $\mathrm{KB}$, participate in multiple cell signal activations and regulate the expression of several inflammatory mediators $(47,48)$. Similarly, the results of the present study indicated that PQ stimulation increased the p-PI3K and p-AKT expression levels, which were downregulated following treatment with LXA4. Since the PI3K/AKT signaling pathway is demonstrated as a key regulator of NF- $\mathrm{KB}$ activation by targeting the transactivation domain of NF- $\mathrm{kB}$ p65 in an IKK $\beta$-dependent manner (49), nuclear NF- $\kappa B$ downregulation may be partly due to the inhibitory effects of LXA4 on the PI3K/AKT pathway.

To further verify the effect of LXA4 on TLR4/ MyD88-mediated NF- $\kappa \mathrm{B} / \mathrm{PI} 3 \mathrm{~K} / \mathrm{AKT}$ signaling pathway, the present study constructed a GV417-TLR4 plasmid and explored the related changes. The results suggested that the enforced overexpression of TLR4 abrogated the protective effects of LXA4, with the emphasis on re-elevated ROS production, re-enhanced pro-inflammatory cytokine levels and re-activated the MyD88 and NF-kB/PI3K/AKT signaling pathways.

In conclusion, the in vitro and in vivo experiments of the present study demonstrated that LXA4 negatively regulated $P Q$-induced inflammation and oxidative stress through the PI3K/AKT/NF- $\kappa B$ pathway mediated by TLR4 and MyD88, possibly providing a novel therapeutic strategy against PQ-induced toxicity. However, in the present study, it was not determined whether other signaling pathways, such as FPR2, HMGB1, MAPK and NLRP3, are also involved in the protective effects of LXA4 on PQ-induced ALI. Therefore, further research is required on the functions of LXA4.

\section{Acknowledgements}

Not applicable.

\section{Funding}

The present study was supported by the Natural Science Foundation of Liaoning Province, China (grant no. 201602879).

\section{Availability of data and materials}

The analyzed datasets generated in the present study are available from the corresponding author upon reasonable request.

\section{Authors' contributions}

YL and MZ performed the majority of the entire research, and together with the other authors, ensure the integrity of the entire research. All authors (YL, NW, ZM, YW, YY, ZZ, YH and MZ) participated in the study concept and the design and the definition of knowledge content of this research. YL, NW and $\mathrm{MZ}$ contributed to the experimental research, data collection and statistical analysis. YL analyzed and interpreted the results and drafted the manuscript. YL, ZM, YW, YY, ZZ and $\mathrm{YH}$ contributed to the writing, review and revision of the manuscript. MZ contributed to the preparation of the final version of manuscript. All authors were responsible for the final content and read and approved the final manuscript.

\section{Ethics approval and consent to participate}

The experiments were approved by the Ethics Committee of Shengjing Hospital of China Medical University (approval no. 2019PS656K). All procedures involving animals were performed in accordance with the ARRIVE guidelines and the National Institutes of Health Guidelines for the Care and Use of Laboratory Animals (NIH Publications no. 8023, revised in 1978).

\section{Patient consent for publication}

Not applicable.

\section{Competing interests}

The authors declare that they have no competing interests.

\section{References}

1. Baltazar T, Dinis-Oliveira RJ, Duarte JA, de Lourdes Bastos M and Carvalho F: Paraquat research: Do recent advances in limiting its toxicity make its use safer? Br J Pharmacol 168: 44-45, 2013.

2. Moon JM, Chun BJ and Cho YS: The characteristics of emergency department presentations related to acute herbicide or insecticide poisoning in South Korea between 2011 and 2014. J Toxicol Environ Health A 79: 466-476, 2016.

3. Sun B and Chen YG: Advances in the mechanism of paraquat-induced pulmonary injury. Eur Rev Med Pharmacol Sci 20: 1597-1602, 2016.

4. Li G, Yuzhen L, Yi C, Xiaoxiang C, Wei Z, Changqing Z and Shuang Y: DNaseI protects against paraquat-induced acute lung injury and pulmonary fibrosis mediated by mitochondrial DNA. Biomed Res Int 2015: 386952, 2015.

5. Liu ZN, Zhao M, Zheng Q, Zhao HY, Hou WJ and Bai SL: Inhibitory effects of rosiglitazone on paraquat-induced acute lung injury in rats. Acta Pharmacol Sin 34: 1317-1324, 2013.

6. Liu Z, Sun M, Wang Y, Zhang L, Zhao H and Zhao M: Silymarin attenuated paraquat-induced cytotoxicity in macrophage by regulating Trx/TXNIP complex, inhibiting NLRP3 inflammasome activation and apoptosis. Toxicol In Vitro 46: 265-272, 2018.

7. Toygar M, Aydin I, Agilli M, Aydin FN, Oztosun M, Gul H, Macit E, Karslioglu Y, Topal T, Uysal B and Honca M: The relation between oxidative stress, inflammation, and neopterin in the paraquat-induced lung toxicity. Hum Exp Toxicol 34: 198-1204, 2015.

8. Chen T, Wang R, Jiang W, Wang H, Xu A, Lu G, Ren Y, Xu Y, Song Y, Yong $\mathrm{S}$, et al: Protective effect of astragaloside IV against paraquat-induced lung injury in mice by suppressing rho signaling. Inflammation 39: 483-492, 2016.

9. Levy BD and Serhan CN: Resolution of acute inflammation in the lung. Annu Rev Physiol 76: 467-492, 2014.

10. Shimizu S, Ogawa T, Seno S, Kouzaki H and Shimizu T: Pro-Resolution mediator lipoxin A4 and its receptor in upper airway inflammation. Ann Otol Rhinol Laryngol 122: 683-689, 2013.

11. Romano M, Cianci E, Simiele F and Recchiuti A: Lipoxins and aspirin-triggered lipoxins in resolution of inflammation. Eur J Pharmacol 5: 49-63, 2015. 
12. Zhang L, Wu P, Jin Sw, Yuan P, Wan Jy, Zhou Xy, Xiong W, Fang F and Ye Dy: Lipoxin A4 negatively regulates lipopolysaccharide-induced differentiation of RAW264.7 murine macrophages into dendritic-like cells. Chin Med J (Engl) 5: 981-987, 2007.

13. Martini AC, Forner S, Bento AF and Rae GA: Neuroprotective effects of lipoxin A4 in central nervous system pathologies. Biomed Res Int 2014: 316204, 2014.

14. Wu L, Li HH, Wu Q, Miao S, Liu ZJ, Wu P and Ye DY: Lipoxin A4 activates Nrf2 pathway and ameliorates cell damage in cultured cortical astrocytes exposed to oxygen-glucose deprivation/reperfusion insults. J Mol Neurosci Aug 56: 848-857, 2015.

15. Zong L, Li J, Chen X, Chen K, Li W, Li X, Zhang L, Duan W, Lei J, Xu Q, et al: Lipoxin A4 attenuates cell invasion by inhibiting ROS/ERK/MMP pathway in pancreatic cancer. Oxid Med Cell Longev 2016: 6815727, 2016.

16. Zhou XY, Wu P, Zhang L, Xiong W, Li YS, Feng YM and Ye DY: Effects of lipoxin A(4) on lipopolysaccharide induced proliferation and reactive oxygen species production in RAW264.7 macrophages through modulation of G-CSF secretion. Inflamm Res 56: 324-333, 2007.

17. Zong H, Li X, Lin H, Hou C and Ma F: Lipoxin A4 pretreatment mitigates skeletal muscle ischemia-reperfusion injury in rats. Am J Transl Res 9: 1139-1150, 2017.

18. Zhao Q, Shao L, Hu X, Wu G, Du J, Xia J and Qiu H: Lipoxin a4 preconditioning and postconditioning protect myocardial ischemia/reperfusion injury in rats. Mediators Inflamm 2013: 231351, 2013

19. Chen XQ, Wu SH, Zhou Y and Tang YR: Lipoxin A4-induced heme oxygenase-1 protects cardiomyocytes against hypoxia/reoxygenation injury via p38 MAPK activation and Nrf2/ARE complex. PLoS One 24: e67120, 2013.

20. Liu ZN, Zhao H, Liu W, Li T, Wang Y and Zhao M: NLRP3 inflammasome activation is essential for paraquat-induced acute lung injury. Inflammation 38: 433-444, 2015

21. Wang Q, Lian QQ, Li R, Ying BY, He Q, Chen F, Zheng X, Yang Y, Wu DR, Zheng SX, et al: Lipoxin A(4) activates alveolar epithelial sodium channel, na, K-ATPase, and increases alveolar fluid clearance. Am J Resp Cell Mol Biol 48: 610-618, 2013.

22. Qi W, Li H, Cai XH, Gu JQ, Meng J, Xie HQ, Zhang JL, Chen J, Jin XG, Tang Q, et al: Lipoxin A(4) activates alveolar epithelial sodium channel gamma via the microRNA-21/PTEN/AKT pathway in lipopolysaccharide-induced inflammatory lung injury. Lab Invest 95: 1258-1268, 2015.

23. Mikawa K, Nishina K, Takao Y and Obara H: ONO-1714, a nitric oxide synthase inhibitor, attenuates endotoxin-induced acute lung injury in rabbits. Anesth Analg 97: 1751-1755, 2003.

24. Shen H, Wu N, Wang Y, Guo F, Chen L, Zhang Z, Jia D and Zhao M: MyD88 gene knockout attenuates paraquat-induced acute lung injury. Toxicol Lett 5: 41-46, 2017.

25. Kayama H, Ramirez-Carrozzi VR, Yamamoto M, Mizutani T, Kuwata H, Iba H, Matsumoto M, Honda K, Smale ST and Takeda K: Class-Specific regulation of pro-inflammatory genes by MyD88 pathways and IкB६. J Biol Chem 290: 4815, 2015.

26. Hazeki K, Nigorikawa K and Hazeki O: Role of phosphoinositide 3-kinase in innate immunity. Biol Pharm Bull 30: 1617-1623, 2007.

27. Androulidaki A, Iliopoulos D, Arranz A, Doxaki C, Schworer S, Zacharioudaki V, Margioris AN, Tsichlis PN and Tsatsanis C: The kinase aktl controls macrophage response to lipopolysaccharide by regulating microRNAs. Immunity 21: 220-231, 2009.

28. Ruse M and Knaus UG: New players in TLR-mediated innate immunity: PI3K and small rho GTPases. Immunol Res 34: 33-48, 2006

29. Rose MS, Smith LL and Wyatt I: Evidence for energy-dependent accumulation of paraquat into rat lung. Nature 22: 314-315, 1974

30. Wang X, Jiao W, Lin M, Lu C, Liu C, Wang Y, Ma D, Wang X, Yin P, Feng J, et al: Resolution of inflammation in neuromyelitis optica spectrum disorders. Mult Scler Relat Disord 27: 34-41, 2019.

31. Börgeson E, Johnson AM, Lee YS, Till A, Syed GH, Ali-Shah ST, Guiry PJ, Dalli J, Colas RA, Serhan CN, et al: Lipoxin A4 attenuates obesity-induced adipose inflammation and associated liver and kidney disease. Cell Metab 7: 125-137, 2015.
32. Liu L, Zhang P, Zhang Z, Hu Q, He J, Liu H, Zhao J, Liang Y, He Z, Li X, et al: LXA4 ameliorates cerebrovascular endothelial dysfunction by reducing acute inflammation after subarachnoid hemorrhage in rats. Neuroscience 1: 105-114, 2019.

33. Lu H, Zhen J, Wu T, Peng A, Ye T, Wang T, Yu X, Vaziri ND, Mohan C and Zhou XJ: Superoxide dismutase mimetic drug tempol aggravates anti-GBM antibody-induced glomerulonephritis in mice. Am J Physiol Renal Physiol 299: F445-F452, 2010

34. Li Y, Liu Y, Peng X, Liu W, Zhao F, Feng D, Han J, Huang Y, Luo S, Li L, et al: NMDA receptor antagonist attenuates bleomycin-induced acute lung injury. PLoS One 5: e0125873, 2015.

35. Kovach MA and Standiford TJ: Toll like receptors in diseases of the lung. Int Immunopharmacol 11: 1399-1406, 2011

36. Oakes JL, O'Connor BP, Warg LA, Burton R, Hock A, Loader J, Laflamme D, Jing J, Hui L, Schwartz DA and Yang IV: Ozone enhances pulmonary innate immune response to a toll-like receptor-2 agonist. Am J Respir Cell Mol Biol 48: 27-34, 2013.

37. Qin C, Zhang B, Zhang L, Zhang Z, Wang L, Tang L, Li S, Yang Y, Yang F, Zhang P and Yang B: MyD88-Dependent toll-like receptor 4 signal pathway in intervertebral disc degeneration. Exp Ther Med 12: 611-618, 2016

38. Liu W, Shan LP, Dong XS and Liu Z: Toll-Like receptor 4 implicated in acute lung injury induced by paraquat poisoning in mice. Int J Clin Exp Med 5: 3392-3397, 2014.

39. Dong XS, Xu XY, Sun YQ, Wei-Liu, Jiang ZH and Liu Z: Toll-Like receptor 4 is involved in myocardial damage following paraquat poisoning in mice. Toxicology 312: 115-122, 2013.

40. Hu R, Xu H, Jiang H, Zhang Y and Sun Y: The role of TLR4 in the pathogenesis of indirect acute lung injury. Front Biosci (Landmark Ed). 2013 Jun 1; 18: 1244-55.

41. Li TT, Ogino S and Qian ZR: Toll-Like receptor signaling in colorectal cancer: Carcinogenesis to cancer therapy. World J Gastroenterol 1: 17699-17708, 2014.

42. Lawrence T. The nuclear factor NF-kappaB pathway in inflammation. Cold Spring Harb Perspect Biol 1: a001651, 2009.

43. Liu P, Zhou YS, Qin YL, Li L, Liu Y, Xu B, Huang K, Ji CC, Lin F, Wang YG, et al: Mechanism of action for oligomeric proanthocyaniclins in pava qnat-induced acute lung injury. Zhonghua Lao Dong Wei Sheng Zhi Ye Bing Za Zhi 20: 818-822, 2017 (In Chinese)

44. Hu X, Shen $\mathrm{H}$, Wang $\mathrm{Y}$ and Zhao M: Liver X receptor agonist TO901317 attenuates paraquat-induced acute lung injury through inhibition of NF- $\mathrm{KB}$ and JNK/p38 MAPK signal pathways. Biomed Res Int 2017: 4652695, 2017

45. Liu Mw, Su Mx, Zhang W, Wang Yq, Chen M, Wang L and Qian Cy: Protective effect of Xuebijing injection on paraquat-induced pulmonary injury via down-regulating the expression of p38 MAPK in rats. BMC Complement Altern Med 16: 498, 2014.

46. Troutman TD, Hu W, Fulenchek S, Yamazaki T, Kurosaki T, Bazan JF and Pasare C: Role for B-cell adapter for PI3K (BCAP) as a signaling adapter linking Toll-like receptors (TLRs) to serine/threonine kinases PI3K/Akt. Proc Natl Acad Sci USA 3: 273-278, 2012

47. Chu AJ: Antagonism by bioactive polyphenols against inflammation: A systematic view. Inflamm Allergy Drug Targets 13: 34-64, 2014.

48. Lo JY, Kamarudin MN, Hamdi OA, Awang K and Kadir HA: Curcumenol isolated from curcuma zedoaria suppresses akt-mediated $\mathrm{NF}-\kappa \mathrm{B}$ activation and $\mathrm{p} 38 \mathrm{MAPK}$ signaling pathway in LPS-stimulated BV-2 microglial cells. Food Funct 6 : 3550-3559, 2015.

49. Madrid LV, Mayo MW, Reuther JY and Baldwin AS Jr: Akt stimulates the transactivation potential of the RelA/p65 Subunit of NF-kappa B through utilization of the Ikappa B kinase and activation of the mitogen-activated protein kinase p38. J Biol Chem 1: 18934-18940, 2001.

This work is licensed under a Creative Commons Attribution-NonCommercial-NoDerivatives 4.0 International (CC BY-NC-ND 4.0) License. 